\title{
Veränderte Kompetenzanforderungen an Mitarbeitende infolge zunehmender Automatisierung - Eine Arbeitsfeldbetrachtung
}

\author{
Michèle Rieth ${ }^{1}(\mathbb{D}) \cdot$ Vera Hagemann ${ }^{1}$ \\ Angenommen: 14. Januar 2021 / Online publiziert: 28. Januar 2021 \\ (c) Der/die Autor(en) 2021
}

\section{Zusammenfassung}

Basierend auf einer Arbeitsfeldbetrachtung im Bereich der Flugsicherung in Österreich und der Schweiz liefert dieser Artikel der Zeitschrift Gruppe. Interaktion. Organisation. (GIO) einen Überblick über automatisierungsbedingte Veränderungen und die daraus resultierenden neuen Kompetenzanforderungen an die Beschäftigten im Hochverantwortungsbereich. Bestehende Tätigkeitsstrukturen und Arbeitsrollen verändern sich infolge zunehmender Automatisierung grundlegend, sodass Organisationen neuen Herausforderungen gegenüberstehen und sich neue Kompetenzanforderungen an Mitarbeitende ergeben. Auf Grundlage von 9 problemzentrierten Interviews mit Fluglotsen sowie 4 problemzentrierten Interviews mit Piloten werden die Veränderungen infolge zunehmender Automatisierung und die daraus resultierenden neuen Kompetenzanforderungen an die Beschäftigten in einer High Reliability Organization dargestellt. Dieser Organisationskontext blieb bisher in der wissenschaftlichen Debatte um neue Kompetenzen infolge von Automatisierung weitestgehend unberücksichtigt. Die Ergebnisse deuten darauf hin, dass der Mensch in High Reliability Organizations durch Technik zwar entlastet und unterstützt werden kann, aber nicht zu ersetzen ist. Die Rolle des Menschen wird im Sinne eines Systemüberwachenden passiver, wodurch die Gefahr eines Fähigkeitsverlustes resultiert und der eigene Einfluss der Beschäftigten abnimmt. Ferner scheinen die Anforderungen, denen sie sich infolge zunehmender Automatisierung gegenüberstehen sehen, zuzunehmen, was in einem Spannungsfeld zu ihrer passiven Rolle zu stehen scheint. Die Erkenntnisse werden diskutiert und praktische Implikationen für das Kompetenzmanagement und die Arbeitsgestaltung zur Minimierung der identifizierten restriktiven Arbeitsbedingungen abgeleitet.

Schlüsselwörter Kompetenzanforderungen · Kompetenzverschiebung · Automatisierung · High Reliability Organization $(\mathrm{HRO}) \cdot$ Motivation · Arbeitsgestaltung

Michèle Rieth, M.Sc

mrieth@uni-bremen.de

Prof. Dr. Vera Hagemann vhagemann@uni-bremen.de
Fachbereich Wirtschaftswissenschaft, Fachgebiet Wirtschaftspsychologie und Personalwesen, Universität Bremen, Enrique-Schmidt-Straße 1, 28359 Bremen, Deutschland 


\title{
Changed competence requirements for employees as a result of increasing automation - a work field view
}

\begin{abstract}
Based on a working field analysis in the area of air traffic control in Austria and Switzerland, this article of the journal Gruppe. Interaction. Organisation. (GIO) provides an overview of automation-related changes and the resulting new competence requirements for employees in High Reliability Organizations. As a result of increasing automation, work processes and job roles are changing fundamentally. Organizations face new challenges and new competency requirements for employees emerge. On the basis of 9 problem-centered interviews with air traffic controllers and 4 problem-centered interviews with pilots, these changes and the new competence requirements for employees in High Reliability Organizations are presented. This organizational context has so far been largely unconsidered in the scientific debate about new competencies. The results indicate that automation can support employees in High Reliability Organizations but cannot replace them. As a result of increasing automation, the role of humans as a system controller becomes more passive. This leads to the risk of skill degradation and reduces the employees' own influence. Despite their passive role, the competence requirements seem to increase which leads to a field of tension. The article concludes with practical implications for a suitable competence management and work design in order to minimize the identified restrictive working conditions.
\end{abstract}

Keywords Competence Requirements · Competence Shift · Automation · High Reliability Organization (HRO) · Motivation · Work Design

\section{Einleitung}

Die Arbeitswelt des 21. Jahrhunderts wird zunehmend durch Digitalisierung und Automatisierung geprägt (Härtwig und Sapronova 2020). Digitalisierung meint dabei die Einführung digitaler Technologien in Unternehmen im Sinne des digitalen Transformationsprozesses (Petry 2019). Sobald einzelne Funktionen oder gesamte menschliche Tätigkeiten auf ein System übertragen werden, wird von Automation oder Automatisierung häufig synonym gesprochen. Der Begriff Automatisierung bezieht sich jedoch auf den Prozess, während Automation das Resultat dieser Verschiebung beschreibt (Hauß und Timpe 2000; Heßler 2019).

Mit Blick auf den öffentlichen und wissenschaftlichen Diskurs herrscht Einigkeit darüber, dass bestehende Tätigkeitsstrukturen und Arbeitsrollen durch Digitalisierung und Automatisierung grundlegend verändert werden. Organisationen stehen neuen Herausforderungen gegenüber und neue Kompetenzanforderungen an Mitarbeitende ergeben sich (Böving et al. 2019; Cascio und Montealegre 2016; Demerouti 2020; Härtwig und Sapronova 2020; Höhne et al. 2017; Hoffman et al. 2014; Umbach et al. 2018). Es reicht nicht aus, den Auf- und Ausbau der Kompetenzen in einer Organisation dem Zufall zu überlassen. Es bedarf einer an den strategischen Zielen der Organisation ausgerichteten Planung, Durchführung und Kontrolle der Kompetenzentwicklung der Beschäftigten (Kauffeld und Paulsen 2018). Im Rahmen eines solchen strategischen Kompetenzmanagements ist es aufgrund der heutigen Schnelllebigkeit unerlässlich, auch zukünftige Anforderungen, die sich z.B. aufgrund steigender Automatisierung ergeben, frühzeitig zu berücksichtigen (Campion et al. 2011; Kauffeld und Paulsen 2018), um wettbewerbsfähig zu bleiben.

Die veränderten Kompetenzanforderungen infolge der Automatisierung werden vor allem im industriellen Bereich bereits vielfach diskutiert (Dworschak et al. 2020; Grzybowska und Łupicka 2017; Hecklau et al. 2016, 2017; Hirsch-Kreinsen 2018; Zajac und Schwede 2017). Vereinzelt wurden die Konsequenzen auch für die Logistik und den Einzelhandel (Böving et al. 2019; Umbach et al. 2018; Windelband et al. 2010) sowie branchenunabhängig (Aepli et al. 2017; DGFP 2016; Hammermann und Stettes 2016; Molina et al. 2018) analysiert. Bisher konnten jedoch keine wissenschaftlichen Studien identifiziert werden, die sich mit den veränderten Kompetenzanforderungen in High Reliability Organizations (HROs) infolge der Automatisierung beschäftigen, woran dieser Beitrag ansetzt.

\subsection{HROs und Automatisierung}

HROs sind Organisationen, die aufgrund ihrer hohen Verantwortung gegenüber anderen Menschen und der Umwelt besonders achtsam und zuverlässig arbeiten müssen. Jeder Fehler kann gravierende Folgen nach sich ziehen. $\mathrm{Zu}$ den HROs zählen bspw. die Polizei, Feuerwehr, Luftfahrt, Kernkraftwerke oder medizinische Versorgung. Sie machen einen großen Anteil der Beschäftigtenstruktur aus (Hagemann 2011). Teams, die in solchen Organisationen arbeiten, werden High Responsibility Teams (HRTs) genannt (Hagemann 2011). In der Literatur lassen sich eine Reihe einzigartiger Eigenschaften von HROs identifizieren (Sutcliffe 2011). Aspekte, wie hochqualifiziertes Personal, häufige Prozessprüfungen und kontinuierliche Verbesserungs- 
Abb. 1 Automationsmodell. (In Anlehnung an Parasuraman et al. 2000)

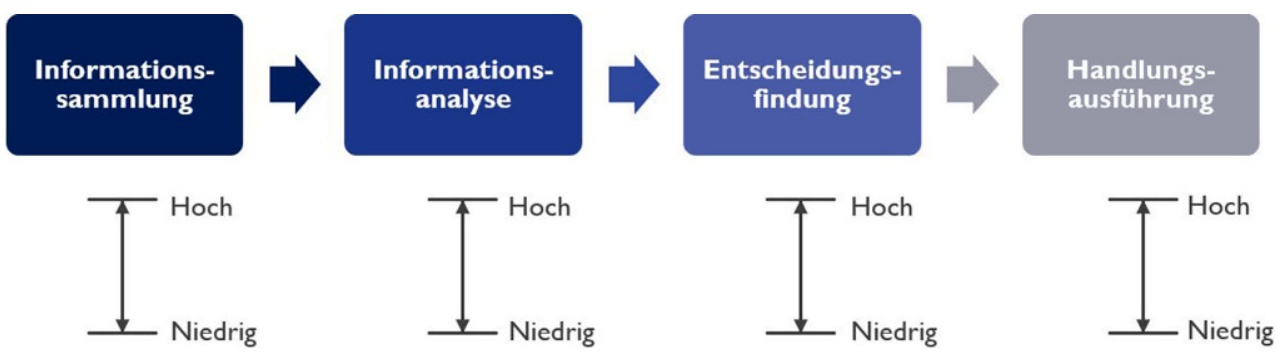

bemühungen (Roberts et al. 2005) sind vereinzelt auch in anderen leistungsstarken Organisationen zu finden. Andere Eigenschaften, wie das organisationsweite stark ausgeprägte Verantwortungsbewusstsein und die damit verbundene Haftbarkeit aller Akteure, das Gefühl der Vulnerabilität, die weit verbreitete Besorgnis über Ausfälle und Fehler sowie die überaus pessimistisch etablierten Vorsichtsmaßnahmen in Bezug auf mögliche Ausfälle und Fehler sind für HROs charakteristisch (Roberts 1990; Roberts und Rousseau 1989; Sutcliffe 2011; Weick und Sutcliffe 2016). Obwohl sie ständig unter schweren Bedingungen arbeiten und oft unerwartete Ereignisse in Sekundenschnelle bei hoher Komplexität bewältigen müssen, treten Unfälle und Störungen weitaus seltener auf, als statistisch zu erwarten wäre. Weick und Sutcliffe (2016) führen diesen Erfolg auf das achtsame Handeln der Akteure (Mindfulness) zurück. Damit beziehen sie sich auf die besondere Organisationsweise von HROs, mit der sie unerwartete Ereignisse rechtzeitig erkennen, deren Weiterentwicklung verhindern oder begrenzen und das System erneut zum Laufen bringen (Weick und Sutcliffe 2016). HROs stellen somit sehr spezifische Anforderungen an ihr Personal und sind nicht vergleichbar mit anderen Organisationen (Hagemann et al. 2012).

HROs arbeiten mit sehr komplexen Technologien (Weick und Sutcliffe 2016). So nimmt die Automatisierung dort einen hohen Stellenwert ein. Gerade in HROs bestehen die Ziele der Automatisierung neben ökonomischen Vorteilen auch in der Erhöhung der Sicherheit und Zuverlässigkeit. Gleichzeitig bergen automatisierte Systeme in HROs neue Risiken, die weitreichende Folgen nach sich ziehen können, z. B. bei fehlerhafter Ausführung oder Ausfall der Automation. In solchen kritischen Momenten muss dann wieder der Mensch aktiv werden und die ursprünglich an die Automation ausgelagerten Funktionen eigenständig ausführen (Manzey 2008). Der Mensch bleibt somit weiterhin eine wichtige Ressource in Organisationen. Seine Rolle verändert sich jedoch grundlegend. Aktive Steuerungsaufgaben nehmen ab, da die Automation diese zunehmend übernimmt. Stattdessen muss der Mensch die Rolle des Systemüberwachenden einnehmen und im Sinne des Supervisory Controls überprüfen, ob die Automation korrekt arbeitet. Somit gewinnen passive Überwachungsaufgaben für den Menschen an Be- deutung (Manzey 2008; Sheridan und Parasuraman 2005; Hoffman et al. 2014).

Eine der größten Herausforderung der Mensch-Maschinen-Interaktion in HROs besteht darin, dass der Mensch in seiner passiven Funktion stets wachsam bleiben, ein angemessenes Situationsbewusstsein aufrechterhalten und im Störfall in der Lage sein muss, einzugreifen (Manzey 2008). Je zuverlässiger jedoch die Automation arbeitet, desto wahrscheinlicher ist ein übersteigertes Vertrauen in sie. In diesem Kontext etablierte sich der Ausdruck Ironies of Automation (Bainbridge 1983). Durch Automation soll der Mensch entlastet werden, das menschliche Intervenieren wird im Störfall jedoch umso herausfordernder, je komplexer die Prozesse und je stärker die Aufgaben automatisiert sind (Bainbridge 1983). Eine weitere Folge der Nutzung automatisierter Systeme besteht im Verlust der notwendigen Fähigkeiten (Parasuraman et al. 2000).

Diese Ausführungen zeigen, dass Automatisierung in HROs kein Alles-oder-Nichts-Phänomen darstellt und ein adäquates Abwägen von Kosten und Nutzen vorausgehen sollte. Die Auswirkungen auf die menschliche Arbeitsleistung hängen davon $a b$, welche und wie viele Funktionen automatisiert werden. Eines der dazu etabliertesten Modelle ist die von Parasuraman et al. (2000) vorgeschlagene Taxonomie der Arten und Stufen von Automation. Die erste Dimension des Modells beschäftigt sich mit der Art der Funktionsübernahme durch die Automation in Anlehnung an den menschlichen Informationsverarbeitungsprozess. Es werden vier aufeinanderfolgende Ebenen unterschieden, die als Informationsaufnahme, Informationsverarbeitung, Entscheidungsfindung und Handlungsausführung bezeichnet werden. Die zweite Dimension beschäftigt sich mit dem Ausmaß der Automatisierung auf jeder dieser vier Ebenen und stellt ein 10-stufiges Kontinuum von niedrig bis hoch dar (vgl. Abb. 1). Mit diesem Ansatz kann jede Art von Automation standardisiert beschrieben und verglichen werden. Dies übertrifft andere Modelle, die häufig nur für eine bestimmte Art von Automation gelten (vgl. Onnasch 2015). Die von Wickens et al. (2010) eingeführte Unterscheidung verschiedener Automatisierungsgrade (degree of automation $=$ DOA) baut auf diesem Modell auf. Der DOA steigt dabei sowohl mit fortschreitender Ebene 
der Art der Informationsverarbeitung als auch mit zunehmender Stufe.

Onnasch et al. (2014) zeigen in ihrer Meta-Analyse, dass zunehmende Automatisierung zu mehr Leistung und weniger Workload seitens der Operateure führt, wenn in der jeweiligen Situation alles planmäßig verläuft. Sobald die Automation jedoch plötzlich ausfällt und der Benutzer nicht in den Prozess hinter der Automation ausreichend einbezogen ist, kann es zu erheblichen Leistungseinbußen kommen. Das Risiko steigt mit zunehmendem DOA. Außerdem stellten sie fest, dass mit zunehmendem DOA das Situationsbewusstsein abnimmt.

Je mehr Unterstützung ein automatisiertes System bietet, desto höher ist das Risiko nachteiliger Auswirkungen auf die Leistung des Menschen und desto größer ist die Wahrscheinlichkeit katastrophaler Folgen, wenn das System ausfällt (Endsley und Kiris 1995; Miller und Parasuraman 2007; Onnasch et al. 2014). Diese negativen Auswirkungen bei Automationsausfall oder -fehlern können darauf zurückgeführt werden, dass der Mensch die hochzuverlässige Automation zum Zeitpunkt des Ausfalls weniger überwacht, ihr zu sehr vertraut (Parasuraman und Riley 1997) und das Situationsbewusstsein verliert (Endsley und Kiris 1995), da die hochgradig (aber nicht perfekt) zuverlässige Automation vor dem ersten Ausfall über einen längeren Zeitraum ordnungsgemäß funktionierte (Parasuraman et al. 1993; Parasuraman und Manzey 2010; Yeh et al. 2003). Dies wird als Complacency Effekt (Parasuraman et al. 1993) oder Automation Bias (Parasuraman und Manzey 2010) beschrieben. Diese Automationsfolgen stellen vor allem in HROs, aufgrund ihrer hohen Verantwortung gegenüber Dritten und der Irreversibilität von Fehlern, ein ernstzunehmendes Risiko dar (Hagemann 2011; Weick und Sutcliffe 2016).

\subsection{Kompetenzbegriff}

Bis heute existiert keine einheitliche Definition des Kompetenzbegriffs (Shippmann et al. 2000; Becker et al. 2018). In Deutschland wird meist die berufliche Handlungskompetenz herangezogen, welche zwischen fachlicher, methodischer, sozialer und personaler Kompetenz differenziert (Becker et al. 2018; Kauffeld 2006; Kauffeld und Paulsen 2018). Per Definition umfasst sie ,alle Fähigkeiten, Fertigkeiten und Wissensbestände, die eine Person, ein Team oder eine Organisation bei der Bewältigung konkreter sowie vertrauter als auch neuartiger Arbeitsaufgaben handlungs- und reaktionsfähig machen und sich in der erfolgreichen Bewältigung konkreter Arbeitsanforderungen zeigen" (Kauffeld und Paulsen 2018, S. 14). Anhand dieser Definition wird deutlich, dass berufsbezogene Kompetenzen mehr umfassen, als nur das bloße „Können“ einer Person. Es geht vielmehr darum, in konkreten Arbeitssituationen durch eigenes „Können“ und „Wissen“ erfolgreich zu handeln (vgl. North 2016). Dieses Kompetenzverständnis erinnert an das im anglo-amerikanischen Raum verwendete System der KSAOs (= „knowledge, skills, abilities, and other characteristics that are needed for effective performance in the job“", Campion et al. 2011, S. 226), welches der vorliegenden Arbeit zugrunde gelegt wird.

\subsection{Ziele der Studie und Forschungsannahmen}

Zusammenfassend sei darauf hingewiesen, dass menschliches Arbeitsvermögen infolge der Automatisierung in HROs nicht unwichtiger wird, sondern das Wissen, die Fertigkeiten und Fähigkeiten der Menschen an Bedeutung gewinnen, da sie im Zusammenspiel mit der Automation nicht nur Routineaufgaben gegenüberstehen, sondern kreativ mit unerwarteten Ereignissen bei hoher Komplexität umgehen müssen. Um weiterhin eine zuverlässige Arbeitsweise der HRTs bei zunehmender Automatisierung gewährleisten zu können, bedarf es Forschung, um den Zusammenhang zwischen technologischen Möglichkeiten und den Folgen für die daraus resultierenden (neuen) Anforderungen an die Beschäftigten analysieren zu können und gestaltend Einfluss zu nehmen, woran der vorliegende Beitrag anknüpft.

Ziel dieser Studie ist es, im Rahmen von teilstrukturierten Interviews den Zusammenhang zwischen automatisierungsbedingten Veränderungen der Arbeit und den daraus resultierenden (neuen) Kompetenzanforderungen für Beschäftigte beispielhaft am Arbeitsfeld der Fluglotsen, als eine typische Vertretergruppe eines HRTs, zu analysieren. Um einen möglichst ganzheitlichen Blick auf das Thema zu erlangen, wurden neben Fluglotsen aus Österreich und der Schweiz auch Piloten befragt, da sie mit Fluglotsen über und mithilfe des Automationssystems eng interagieren und dadurch einen realitätsnahen Einblick in deren Arbeitsweisen vor dem Hintergrund zunehmender Automatisierung haben.

Obwohl in HROs immer mehr Technologien eingeführt werden, die die Beschäftigten entlasten und unterstützen sollen, vermuten wir, dass die Kompetenzanforderungen in dieser Branche infolge zunehmender Automatisierung in Anlehnung an Erkenntnisse aus der Industriesoziologie (z.B. Böving et al. 2019; Härtwig und Sapronova 2020; Hirsch-Kreinsen 2018; Windelband et al. 2010) insgesamt vielmehr steigen als abnehmen. Gerade in HROs ist es nicht ausreichend, sich lediglich auf die Technik zu verlassen. Aufgrund der hohen Verantwortung bedarf es weiterhin eines kritischen Mitdenkens von Menschen, die im Falle von Systemfehlern oder -ausfällen jederzeit eingreifen können. Somit nehmen wir ersten an, dass die Grundanforderungen an den Beruf des Fluglotsen bestehen bleiben. Hinzu kommt jedoch die Anforderung, die Systeme und die damit 
verbundenen Systemprozesse zu verstehen und bedienen zu können. Somit nehmen wir zweitens an, dass die Anforderungen insgesamt steigen. Zudem erwarten wir drittens, aufgrund der neu entstandenen Rolle des Menschen als Systemüberwachenden, eine Kompetenzverschiebung von aktiven Steuerungsaufgaben hin zu passiven Überwachungsaufgaben. Basierend auf diesen Annahmen wird davon ausgegangen, dass es einer Anpassung der Kompetenzprofile der Erwerbstätigen in HROs bedarf.

\section{Methode}

Um herauszufinden, welchen Veränderungen und neuen Kompetenzanforderungen Beschäftigte in HROs infolge zunehmender Automatisierung gegenüberstehen, wurde zunächst das dafür genutzt Arbeitsfeld der Fluglotsen aus wissenschaftlicher und praktischer Perspektive umfassend betrachtet. Diese Hochverantwortungsgruppe stellt ein passendes Sampling dar, da die Flugsicherung als eine typische Vertretergruppe einer HRO gilt und als Pionier im Einsatz neuer Technologien betrachtet wird (Eurocontrol o.J.). Es wurde ein subjektbezogener Ansatz gewählt, wobei die Beschäftigten als ExpertInnen ihrer eigenen Tätigkeit betrachtet werden.

\subsection{Stichprobe}

Es wurden neun problemzentrierte Interviews nach Witzel (2000) mit Fluglotsen aus Österreich $(n=3)$ und der Schweiz $(n=6)$ geführt, welche mit I1 bis I9 abgekürzt werden. Das durchschnittliche Alter betrug 36,44 Jahre $(S D=4,59)$. Es nahmen eine Frau und acht Männer teil. Die durchschnittliche Berufserfahrung lag bei 10,8 Jahren $(S D=4,78)$.

Um einen möglichst holistischen Einblick in das Thema zu erhalten, fanden zusätzlich vier weitere Interviews nach der gleichen Methodik mit Piloten aus Deutschland $(n=2)$, Österreich $(n=1)$ und der Schweiz $(n=1)$ statt. Die Piloteninterviews werden mit P1 bis P4 abgekürzt. Das durchschnittliche Alter der Piloten betrug 44,25 Jahre $(S D=9,74)$. Es nahmen vier Männer teil. Die durchschnittliche Berufserfahrung lag bei 22,00 Jahren $(S D=12,43)$. Das Heranziehen der Piloten liegt darin begründet, dass die Interaktion zwischen Lotsen und Piloten über und mithilfe des Automationssystems ein zentraler Bestandteil der Arbeitstätigkeiten beider Berufsgruppen ist. Das Automationssystem stellt eine wichtige Schnittstelle dar. Es unterstützt sie durch entsprechende Funktionen und wird immer häufiger zur wechselseitigen Kommunikation via Data Link herangezogen. Dadurch haben Piloten einen realitätsnahen Einblick in die (veränderten) Arbeitsweisen der Fluglotsen und können die Auswirkungen zunehmender
Automatisierung für ihre LotsenkollegInnen arbeitsplatznah wahrnehmen. Sie stellen eine sinnvolle Ergänzung der subjektiven Lotsenperspektive dar.

\subsection{Interviewdurchführung}

Die Interviews wurden von Juli bis September 2019 mithilfe eines halbstandardisierten Leitfadens telefonisch durchgeführt. Um einen einfachen Einstieg in das Interview zu gewährleisten, wurden zunächst berufsbezogene demographische Fragen zur Person gestellt. Um unsere Forschungsannahmen zu überprüfen, wurden dann die erlebten Veränderungen durch zunehmende Automatisierung am Arbeitsplatz thematisiert, welche unter anderem anhand der vier Stufen des menschlichen Informationsverarbeitungsprozesses in Anlehnung an das Modell von Parasuraman et al. (2000) erfasst wurden (vgl. Abb. 1). Die Befragten wurden dabei explizit gebeten, ihre heutige Arbeitstätigkeit unter Verwendung der technischen Systeme und die damit verbundenen Veränderungen auf jeder Stufe im Vergleich zu früher, als noch manuell mit Papierstreifen gearbeitet wurde (vgl. Abschn. 3.1), darzulegen (z. B. für Stufe 2 - Informationsanalyse: Wie analysierten Sie früher mit Papierstreifen die Informationen aller relevanten Faktoren in Ihrem Sektor? Wie machen Sie dies heute mithilfe des technischen Systems? Was hat sich dadurch für Sie verändert?). Dabei wurden auch die wahrgenommenen Vorteile und Herausforderungen dieser Automatisierung erfasst. Im zweiten Block wurden die veränderten Anforderungen, denen sich Fluglotsen aufgrund zunehmender Automatisierung gegenübersehen, erfragt (z. B. Was muss ein Fluglotse heute bei zunehmender Automatisierung im Vergleich zu früher können?). Im dritten Block wurde nach unterstützenden Aspekten im Umgang mit Automation und möglichen zukünftigen $\mathrm{Au}$ tomatisierungsentwicklungen der Branche gefragt und die damit einhergehenden Ängste und Wünsche erfasst. Der Pilotenleitfaden unterscheidet sich inhaltlich insofern, als dass die Piloten gebeten wurden, nicht für ihre eigene Berufsrolle $\mathrm{zu}$ antworten, sondern die Veränderungen in der Interaktion mit ihren LotsenkollegInnen infolge der Automatisierung zu thematisieren. Die Lotseninterviews dauerten im Schnitt 53 min und variierten zwischen 44 und $80 \mathrm{~min}$. Die Piloteninterviews dauerten durchschnittlich 44 min und variierten zwischen 30 und $58 \mathrm{~min}$.

\subsection{Auswertung}

Die Interviews wurden transkribiert und mithilfe des Programms MAXQDA im Sinne der strukturierenden qualitativen Inhaltsanalyse (Mayring und Fenzl 2014) kodiert und ausgewertet. Hierbei wurde eine Kombination von induktivem und deduktivem Vorgehen gewählt. So wurden anhand der Literatur und des Leitfadens deduktiv erste Kategorien 


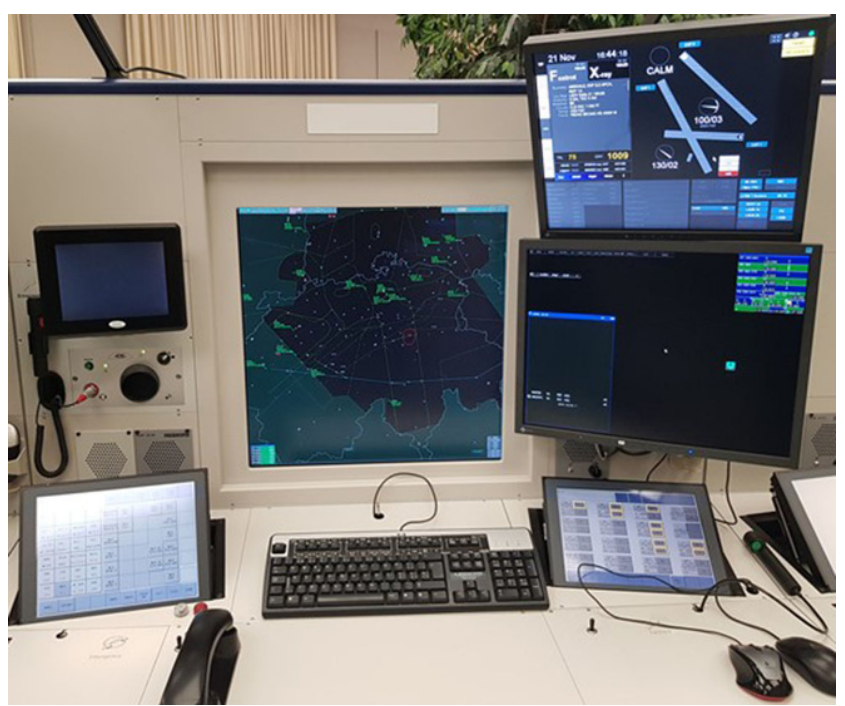

Abb. 2 Der Arbeitsplatz eines Fluglotsen. (Foto aufgenommen und frei zur Verfügung gestellt von Tino Balk, Skyguide Flugverkehrsleiter Überflugkontrolle Dübendorf am 05.12.2019)

gebildet, denen das Textmaterial zugeordnet werden sollte. Im Laufe der Auswertung wurden diese durch induktiv neue Kategorien anhand des Interviewmaterials ergänzt. Für jede Kategorie wurde eine Definition, typische Beispieltextpassagen und Kodierregeln zur Abgrenzung zwischen den Kategorien festgelegt. Die identifizierten Kategorien wurden schließlich vor dem Hintergrund der Forschungsannahmen inhaltlich reflektiert.

\section{Ergebnisse}

Anhand der Erkenntnisse der Interviews sowie der wissenschaftlichen und praktischen Arbeitsfeldbetrachtung werden die Arbeitstätigkeiten der Fluglotsen zunächst zusammenfassend präsentiert. Fluglotsen beaufsichtigen und navigieren den Luftverkehr in einem zugewiesenen Sektor mithilfe von modernen Technologien im Zweierteam (ZweiAugen-Prinzip) und sorgen für einen sicheren und geordneten Verkehrsablauf. Sie sind für die Einhaltung des nötigen örtlichen und zeitlichen Abstands zwischen den Fliegern verantwortlich. Dabei stehen sie in engem Kontakt $\mathrm{zu}$ (Team)KollegInnen der eigenen und angrenzenden Sektoren, PilotInnen und Flughafengesellschaften (Berufenet 2019). Abb. 2 stellt beispielhaft den Arbeitsplatz eines Fluglotsen dar. Ein Blick ins O*NET (2019) zeigt, welch breites Kompetenzprofil der Beruf des Fluglotsen schon heute erfordert. So bedarf es bspw. einem ausgeprägten räumlichen Vorstellungsvermögen, guten analytischen Fähigkeiten und einer überdurchschnittlichen Problemlösekompetenz, um nur ein paar wenige Aspekte zu benennen. Diese werden im Folgenden als Grundanforderungen bezeichnet und beziehen sich auf die Basiskompetenzen, die ein Fluglotse unabhängig von der Technologie für die Ausübung seines Jobs mitbringen muss. Bezieht man nun die stetig fortschreitende Automatisierung der Branche ein, so stellt sich die Frage, welche neuen oder veränderten Kompetenzanforderungen daraus resultieren.

\subsection{Stand der Automatisierung}

Referenzpunkt für die Automatisierung stellt das damalige manuelle Papierstreifensystem der Flugsicherung dar. Dabei existierte zwar schon ein Radarsystem, welches jedoch nur zur reinen Luftlage-Darstellung mit Verzögerung, Ungenauigkeiten und ohne Flightdata Processing genutzt werden konnte. Sämtliche Flugdaten der Flugzeuge wurden in tabellarischer Form auf je einem ausgedruckten Papierstreifen dargestellt. Ein Fluglotse erhielt nach und nach alle Papierstreifen der durch seinen Sektor fliegenden Flugzeuge. Jeder Flugstreifen wurde in einem Plastikhalter auf Streifenbretter gelegt und der Situation entsprechend angeordnet. Mit einem Stift konnte das Lotsenteam alle relevanten Informationen, wie z.B. Freigaben oder aktualisierte Flugzeiten, handschriftlich nach standardisierten Regeln auf den Streifen notieren (SKYbraby 2019). Die Lotsen waren kognitiv sehr gefordert. Sie mussten den Überblick behalten, ohne jegliche Art technischer Unterstützung. Das manuelle Papierstreifensystem wurde in der Schweiz durch ein digitales Streifensystem abgelöst (Steinke 2013), bei dem alle Flugdaten auf digitalen Streifen im System hinterlegt sind. Die Streifen sind mit einem Sicherheitssystem verknüpft, welches Warnsignale für Konflikte liefert, Änderungen auf den Streifen werden digital vorgenommen und automatisch über das System an angrenzende Sektoren übermittelt (SKYbraby 2019). In Österreich wurde das Papierstreifensystem durch ein automatisiertes streifenloses System ersetzt. Sämtliche Daten, die früher auf Streifen standen, werden in das Radarsystem integriert und können am Monitor abgelesen werden. Die Flugdaten sind dabei verknüpft, die Lotsen werden automatisch über potenzielle Konflikte zwischen Fliegern informiert und erhalten Vorschläge zur optimalen Lande-/Anflugreihenfolge. Die Koordination zu Nachbarsektoren und PilotInnen kann automatisch über das System erfolgen (LuftfahrtMagazin 2015). Die Systeme werden immer wieder durch genauere und aktuellere Automationsfunktionen versehen.

Der aktuelle Automationsgrad im Vergleich zum früheren wurde im Rahmen der Interviews in Anlehnung an die von Parasuraman et al. (2000) entwickelte Taxonomie analysiert (vgl. Abb. 3). Der Referenzpunkt der Automatisierung, das damalige Papierstreifensystem, ist in Abb. 3 durch einen Kreis gekennzeichnet und liegt auf allen vier Stufen gemäß der Klassifizierung nach Parasuraman et al. (2000) auf einem sehr niedrigen Level, da das System keine auto- 


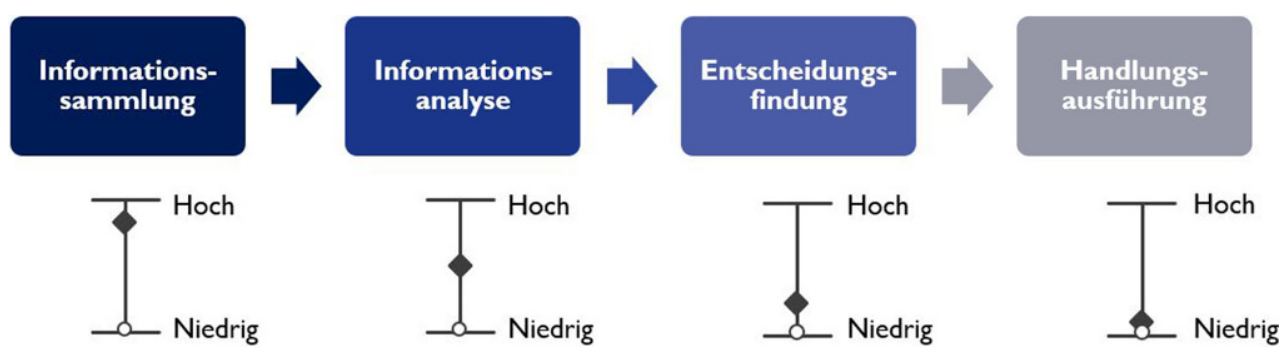

Abb. 3 Vereinfachte Darstellung zum Stand des heutigen Automationsgrades (Position der Rauten) in der Flugsicherung im Vergleich zum früheren manuellen Papierstreifensystem (Position der Kreise), identifiziert anhand der Interviews. (In Anlehnung an Parasuraman et al. 2000)

matisierten Unterstützungsfunktionen lieferte. Die Rauten zeigen den heutigen Automationsgrad auf jeder Stufe, abgeleitet aus den Interviewaussagen. Die Informationssammlung, die erste Stufe des Modells, wurde in der Flugsicherung heute im Vergleich zu früher am stärksten automatisiert. Daher ist die Raute in Abb. 3, gemäß der Klassifizierung nach Parasuraman et al. (2000), auf einem sehr hohen Level. Die Fluglotsen sitzen vor verschiedenen Monitoren, worauf ihnen hochautomatisiert alle Informationen, die sie für ihre Arbeit benötigen, wie z.B. alle relevanten Flieger, Flugrouten sowie Wind- und Wetterlagen, zusammenfassend präsentiert werden. Die Befragten berichten: „Sammeln ist eigentlich ein bisschen übertrieben. Es wird mir ja eigentlich alles [vom System] dargestellt“(I5). Jedoch müssen sie für eine solide Informationssammlung das System stets beherrschen: „Wenn man weiß, wie man es bedienen muss, dann kann man alles finden, was man braucht" (I5).

Die zweite Stufe, die Informationsanalyse, ist gemäß der Einteilung nach Parasuraman et al. (2000) moderat automatisiert. Die Analyse liegt in der Flugsicherung noch stärker beim Menschen, jedoch wird dieser durch hochautomatisierte Tools unterstützt. So bekommen die Lotsen bspw. Konflikte zwischen Fliegern optisch und akustisch angezeigt. Die Interviewten sind sich einig, dass diese Hilfsmittel lediglich als Unterstützungstools fungieren und die menschliche Analyse nicht ersetzen, da sie nicht immer korrekt funktionieren: „Es ist auch so definitiv bei uns festgeschrieben, dass das System [...] als reines Informationstool dient. Also wir sind uns da sehr wohl bewusst, dass das nicht ganz 100-prozentig immer richtig ist" (I2).

Der Automationsgrad auf der Stufe der Entscheidungsfindung fällt in der Flugsicherung gering aus. Es gibt bisher keine Systeme, die eigenständig eine Entscheidung treffen. Die Lotsen erklären, dass sie zwar Unterstützungstools haben, die helfen können, eine gute Entscheidung zu finden, wie z.B. eine Erprobungsfunktion. Mit dieser kann vorab überprüft werden, welche Konsequenzen eine Entscheidung hätte, wenn man sie ausführen würde, wie bspw. das Flight Level eines Fliegers ändern und überprüfen, wie dieser sich zu anderen Fliegern im gleichen Sektor verhalten würde. Die finale Entscheidungsfindung liegt jedoch wei- terhin beim Menschen: „Da ist dann wirklich der Mensch führend" (I8).

Auf der letzten Stufe, der Handlungsausführung, liegt der geringste Automationsgrad vor. Absprachen mit PilotInnen und Nachbarsektoren geschehen dabei zwar im Vergleich zum Papierstreifensystem weniger verbal und immer mehr digital über Data Link oder Systemeingaben, die Umsetzung einer getroffenen Entscheidung liegt jedoch alleine beim Menschen.

Zukünftige Automationsschritte zielen vor allem auf die Informationsanalyse und Entscheidungsfindung ab. So soll die Konflikterkennung genauer und zuverlässiger automatisiert werden und Tools, die Lösungsvorschläge zur Navigation der Flugzeuge in einem Sektor machen, entwickelt werden.

\subsection{Die Rolle des Menschen am Arbeitsplatz}

Durch zunehmende Automatisierung verändert sich die menschliche Rolle grundlegend. Die Befragten sind sich einig, dass der Mensch trotz Automation als „kritischer Mitdenker" (z.B. P1) auch in Zukunft in HROs benötigt wird und sich die Grundanforderungen an ihren Beruf nicht verändern: „Von den Fähigkeiten an sich benötigen wir meiner Meinung nach noch dieselben wie vorher" (I3). Alle Befragten berichten jedoch, dass sie zunehmend die Funktion des Systemüberwachenden einnehmen und ihre Aufgaben passiver werden: „Immer mehr und mehr Sachen werden vom System übernommen, d.h. man wird mehr vom Aktiv-Controller zum Monitoring-Controller, dass man mehr Überwacher ist als aktiv am Kontrollieren“ (I9). Die Befragten weisen auf die daraus resultierende Gefahr des Fähigkeitsverlustes hin, welcher in Ausfallszenarien fatale Folgen in HROs haben kann: „Die Technik entlastet so stark, dass man sehr von [ihr] abhängig wird. Und es kann durchaus mal sein, dass so ein System, ein Radarbild oder ein Flugplandatensystem z.B., einfach kurze Zeit aussteigt. Und da dann wieder ganz nach Old School zu arbeiten, ist sehr schwierig, weil wir trainieren das sehr wenig. [...] Das ist dann sofort sehr, sehr gefährlich [...]“" (I8). Dies zeigt sich vor allem im Gegensatz zur früheren, 
Abb. 4 Vereinfachte Darstellung des Spannungsfeldes (schraffierte Fläche) zwischen den insgesamt gestiegenen Anforderungen an das Personal und den reduzierten aktiven Anwendungsmöglichkeiten infolge zunehmender Automatisierung. (Die Abbildung dient lediglich der vereinfachten Darstellung und basiert nicht auf quantitativen Daten. Die Grafik ist auf einer inhaltlichen Ebene und nicht auf einer mathematischen Ebene zu interpretieren.)

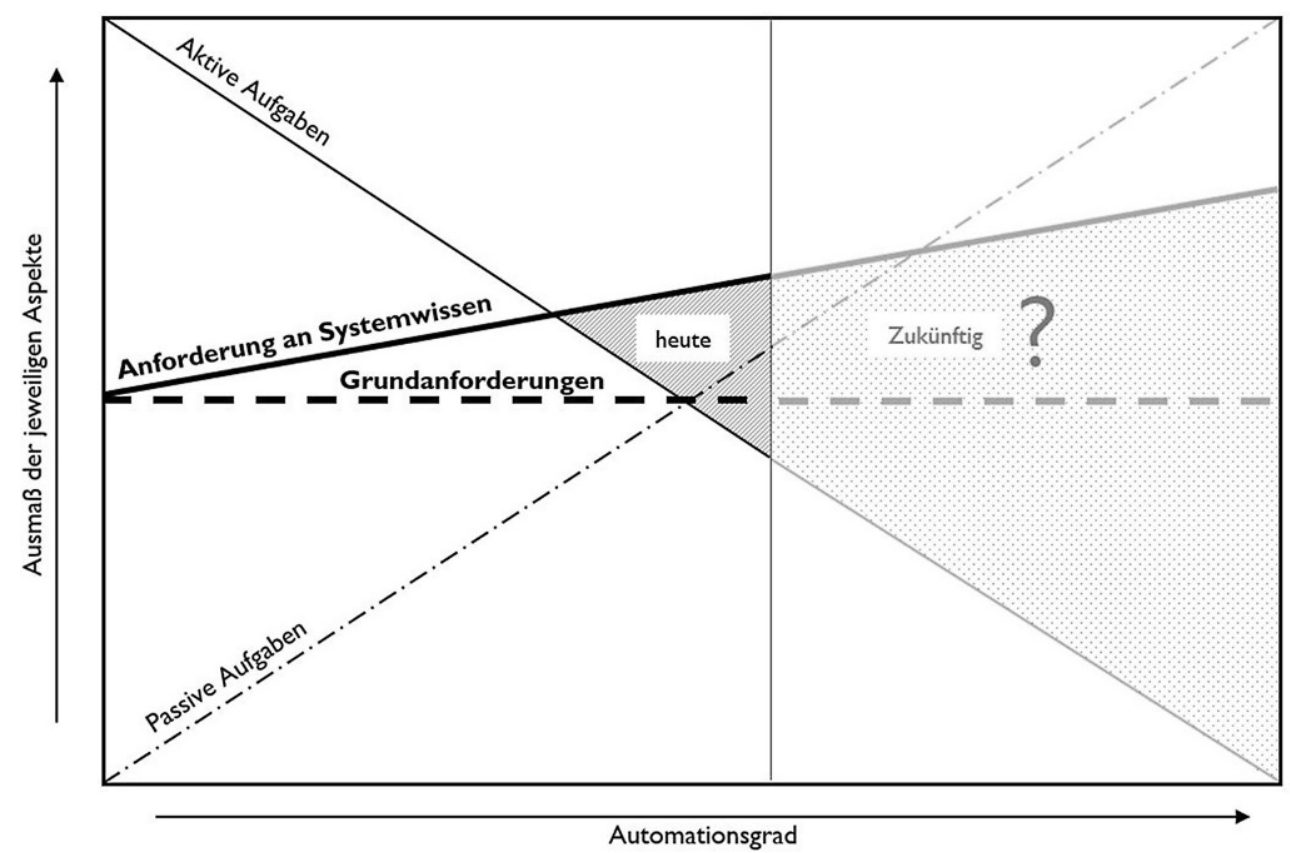

weniger automatisierten Arbeitsweise: ,[...] früher hatte ich die Streifen, das ist irgendwie etwas zum Angreifen, da habe ich wenigstens noch irgendwas, aber das ist jetzt nicht mehr der Fall“" (I3). Darüber sind sich die Lotsen, als auch die Piloten (z. B. P1 und P3) bewusst.

Die Lotsen berichten, dass mit zunehmender Automatisierung eine Standardisierung einhergeht, wodurch sie sich in ihrer Arbeitsfreiheit eingeschränkt fühlen: „Diese $\mathrm{Au}$ tomatisierung und die damit verbundene Standardisierung [hat] dazu geführt, dass die Kreativität abgenommen hat. [...] diese ursprüngliche Arbeit, dieses Hochkreative, diese Motivation, aus diesem gesamten Gewirr an hunderten Flugzeugen etwas zu machen, ist jetzt dem System gewichen“(I1). Die neue Art des Arbeitens wird auch als „Fließbandarbeit" (z. B. I1 und I4) bezeichnet, um damit die einhergehende Standardisierung und die Einschränkung der eigenen Arbeitsfreiheit und des eigenen aktiven Beitrags zu beschreiben. Die Piloten bestätigen dies (z. B. P2).

Dadurch, dass die Befragten in ihrer täglichen Arbeit zunehmend mit einem System interagieren müssen, entsteht eine neue Aufgabe für sie: „Ich muss das System mehr verstehen, wie das System denkt, damit ich das System bedienen kann. [...] Das war früher gar nicht so" (I4). So berichten die Lotsen, dass sie stets die Systemlogik korrekt berücksichtigen müssen (I2) und sich jederzeit bewusst darüber sein müssen, was das System leisten kann, wo die Grenzen dessen sind, welche Auswirkungen ihr Handeln auf das System und die automatisch übermittelten Informationen an angrenzende Sektoren hat (I7). Sie benötigen dazu ein gewisses „Verständnis für die Technik“ (I1). Dies meint keine Informatikfähigkeiten (I5), sondern die Fähig- keit, „ein System zu begreifen“ (I9) und „damit umgehen“ (I1) zu können. Es bedarf also einem Verständnis dafür, „wie das System funktioniert“ (I2). Es ist nötig, ,die Logik hinter [dem] System zu verstehen“ (I2) und „Systemwissen“(I4) zu erlernen. Dies wird als ein Mehraufwand (z.B. I1 und I4) wahrgenommen.

Hinzu kommt ,eine gewisse Form an Systemadministratortätigkeiten. Also wenn das Computersystem nach einer gewissen Art und Weise arbeiten soll, muss man es ja auch dazu bringen, das zu tun (I1)“. Dies wird auch als „Systembefriedigung“ (z.B. I4), „System Maintenance“ (I2) oder „System füttern“ (z. B. I6) bezeichnet und meint, dass das System gewisse „Inputs“ (z. B. I5) benötigt, um korrekt arbeiten zu können. Diese beiden neuen Aufgaben bezeichnen wir im Folgenden zusammenfassend als Systemwissen.

Die Lotsen berichten zudem von einem „Eigenleben des Systems" (I2) und erklären, dass sie Systemmeldungen teilweise nicht nachvollziehen können (I1: „Was will das System jetzt von mir?"). Zudem liefert die Automation scheinbar häufig Fehlalarme, welche als „Normal System Behavior" (I2) abgetan werden. Diese kosten die Lotsen in ihrer Tätigkeit viel Zeit und Kapazität (I7). Sie müssen abwägen, ob sie sich die Fehlalarme tatsächlich alle anschauen oder sich auf ihr eigenes Scanning verlassen (I2). Daher wird gelehrt, dass die Tools nur reine Informationstools sind und der Lotse weiterhin seine eigene Analyse machen muss (I2). Dies zeigt erneut, dass die Grundanforderungen trotz $\mathrm{Au}-$ tomatisierung bestehen bleiben.

Anhand der Interviewergebnisse lassen sich somit, in Übereinstimmung mit unseren Forschungsannahmen, vier Tendenzen erkennen, die in Abb. 4 vereinfacht dargestellt 
sind. Erstens bestätigen sie, dass der Mensch in HROs durch Technik zwar entlastet und unterstützt werden kann, aber auf keiner Automationsstufe zu ersetzen ist. Zweitens bleiben die Grundanforderungen an den Beruf des Fluglotsen somit bestehen (fettgedruckte gestrichelte Linie). Drittens zeigt sich, dass die Anforderungen insgesamt steigen, da die Anforderung, die Systeme zu verstehen und bedienen zu können, hinzukommt (fettgedruckte durchgezogenen Linie). Viertens wird deutlich, dass, aufgrund der neu entstandenen Rolle des Menschen als Systemüberwachenden, eine Kompetenzverschiebung von aktiven Steuerungsaufgaben (dünne durchgezogene Linie) hin zu passiven Überwachungsaufgaben (dünne Strichpunktlinie) erfolgt. Daraus konnten wir anhand dieser Studie für das Personal heute ein erstes Spannungsfeld (schraffierte Fläche) zwischen den insgesamt steigenden Anforderungen und der reduzierten Möglichkeit, die eigenen KSAOs aktiv anzuwenden, erkennen. Der Bereich, in dem sich die Fluglotsen heute bewegen, abhängig von der konkreten Systemausgestaltung am jeweiligen Standort, ist in Abb. 4 vereinfacht dargestellt (schraffierte Fläche) und zeigt, dass passive Aufgaben allmählich Oberhand erhalten (z.B. I2, I7, I9). Ob sich dies zukünftig in diesem Ausmaß weiterentwickeln wird (angedeutet durch gepunktete Fläche), wird im nächsten $\mathrm{Ab}$ schnitt diskutiert.

\section{Diskussion}

Im Rahmen dieser Studie konnten wir zeigen, dass Beschäftigte in HROs unabhängig vom Automationsgrad mit den gleichen Grundanforderungen benötigt werden. Ein Grund dafür könnte die hohe Verantwortung sein, die sie gegenüber anderen tragen. Es reicht, im Gegensatz zu anderen Branchen, nicht aus, sich gänzlich auf die Technik zu verlassen, denn jeder Fehler und Systemausfall kann gravierende Folgen nach sich ziehen. Arbeitet ein automatisiertes System bspw. in der Logistik fehlerhaft, so entstehen dabei eher wirtschaftliche Nachteile aufgrund fehlerhafter Arbeitsabläufe. In HROs kann dieses Risiko nicht eingegangen werden, da Systemfehler oder -ausfälle dazu führen können, dass katastrophale Umweltschäden entstehen oder Menschen sterben, wie z.B. beim Flugzeugabsturz bei Überlingen am Bodensee im Juli 2002, bei dem alle 71 Insassen ums Leben kamen (Bundesstelle für Flugunfalluntersuchung 2004). Die Beschäftigten in HROs müssen trotz Automation jederzeit in der Lage sein, einzugreifen und die ursprünglich an das System ausgelagerten Funktionen wieder eigenständig auszuführen. Es bedarf weiterhin einem kritisch mitdenkenden Menschen mit einem aktuellen Situationsbewusstsein (Endsley und Kaber 1999) und einer hohen Cognitive Readiness (Fletcher 2004). Neben diesen weitestgehend gleichbleibenden Grundanforderungen kann insgesamt jedoch von einem Anforderungsanstieg ausgegangen werden, da die Beschäftigten nun zusätzlich Systemwissen benötigen. Dieses fehlte dem verantwortlichen Lotsen des Flugzeugabsturzes bei Überlingen, dem die Auswirkungen eines bestimmten Systemmodus nicht bewusst waren (Bundesstelle für Flugunfalluntersuchung 2004).

Der Wandel der menschlichen Rolle von einer aktiven in eine passive Rolle stimmt mit der in der Literatur beschriebenen Supervisory Control (Manzey 2008; Sheridan und Parasuraman 2005; Hoffman et al. 2014) überein. Die Ergebnisse unserer Studie zeigen jedoch, dass daraus ein erstes Spannungsverhältnis zu dem insgesamt identifizierten Anforderungsanstieg (vgl. schraffierte Fläche in Abb. 4) resultiert: Auf der einen Seite muss das Personal in HROs trotz Automation weiterhin hochqualifiziert sein und die gleichen KSAOs mitbringen. Auf der anderen Seite wird die Rolle der Beschäftigten immer passiver, wodurch die KSAOs der Beschäftigten immer seltener aktiv zum Einsatz kommen. Dieses Spannungsverhältnis kann erklären, warum seitens der Befragten das Gefühl entsteht, in der eigenen, kreativen Arbeitsfreiheit eingeschränkt zu sein und dass der eigene Beitrag zur Arbeitstätigkeit sinkt. Veranschaulichen wir uns dieses Phänomen einmal mit einem Beispiel: Stellen wir uns vor, ein Berater sitzt hinter einer Glaswand und beobachtet eine Künstliche Intelligenz (KI), die automatisiert auf Grundlage von Algorithmen einen Klienten berät. Der Berater muss kontrollieren, ob die KI korrekte Ratschläge liefert. Dazu muss er weiterhin mitdenken und die Situation des Klienten genauso analysieren wie auch zuvor ohne KI. Er benötigt also die gleichen KSAOs. Zusätzlich muss er, für den Fall, dass er eingreifen muss, die Logik der KI verstehen und wissen, wie er sie zu bedienen hat. Insgesamt steigen also die Anforderungen an den Berater. Doch nun darf er nur noch dann selbst aktiv werden, wenn die KI Fehler macht oder ausfällt. D. h., dass der Berater sein eigenes Wissen, seine Fähigkeiten und Fertigkeiten weitestgehend zurückhalten muss und nur noch selten aktiv einbringen kann. Dass dies negative Auswirkungen auf die Motivation und die Arbeitszufriedenheit haben kann, dürfte nicht verwunderlich sein.

Ausgehend von der Selbstbestimmungstheorie nach Ryan und Deci (2000) ist bekannt, je weniger eine Arbeitstätigkeit selbstbestimmt und autonom ausgeführt wird, desto geringer ist die intrinsische Motivation und umso größer die Gefahr der Demotivation. Auch mit Bezug auf das Job Characteristics Model von Hackman und Oldham (1976) kann angenommen werden, dass unter diesen restriktiven Arbeitsbedingungen die Bedeutsamkeit und die Ganzheitlichkeit der Arbeitsaufgabe verloren gehen, was fatale Folgen für die Motivation, die Arbeitsbefriedigung und die Qualität der Arbeit nach sich ziehen kann. Doch gerade in HROs, in denen die Ausbildung der für den Beruf notwendigen Kompetenzen einen hohen zeitlichen und qualitati- 
ven Stellenwert einnimmt, sollte diesen Aspekten eine hohe Bedeutung zukommen, um weiterhin eine zuverlässige Arbeitsqualität gewährleisten zu können und einer hohen Abwesenheit und Fluktuation entgegenzuwirken. Es stellt sich also die Frage, wie die Verschiebung von einer aktiven in eine stark passive Arbeitsrolle für die weiterhin hochqualifizierten Beschäftigten in HROs auch unter restriktiven Bedingungen erfüllend gestaltet werden kann bzw. wie diese Restriktionen aufgelöst werden können. Dieser Fragestellung soll in Folgestudien nachgegangen werden. Dabei können z.B. die von Hassenzahl et al. (2010) identifizierten Strategien zur Aufrechterhaltung von Wohlbefinden und Motivation im Umgang mit Technik oder die Empfehlungen zur Arbeitsgestaltung nach Parker und Grote (2020) herangezogen werden.

Die Lotsenaussagen bzgl. des „Eigenlebens des Systems" und häufiger Fehlalarme zeigen, dass die heutige Automation noch nicht ausgereift genug ist, um auf den Menschen als Back-Up-Lösung zu verzichten. Es scheint, als befinde man sich momentan in einer Art Übergangsphase, in der das Verhältnis von Automation und menschlicher Tätigkeit ungünstig zu sein scheint. Wird in der Branche wie bisher weiter automatisiert, so besteht die Gefahr des Anstiegs dieses Spannungsfelds (vgl. Abb. 4 gepunktete Fläche), was in HROs, wie oben aufgezeigt, fatal Folgen haben kann. So sollte bei zukünftigen Automatisierungsschritten das identifizierte Spannungsfeld für Fluglotsen reduziert werden. Dies könnte z.B. durch eine menschengerechtere Aufgabenverteilung zwischen System und Mensch gelingen, wobei der Mensch weiterhin einen ausreichend aktiven Part einnehmen kann. Eine Reduzierung des Spannungsfeldes kann auch durch zuverlässigere und weiter automatisierte Systeme erzielt werden, bei denen der Mensch aus der momentanen stark passiv geprägten Monitoringrolle herausgenommen werden kann und die Anforderungen an Systemwissen reduziert werden können. Dann sind auch höhere Automationsniveaus, wie es Parasuraman et al. (2000) vorschlagen, denkbar. Die konkrete Arbeitsgestaltung entscheidet folglich darüber, ob das Spannungsfeld zukünftig größer wird, bestehen bleibt oder reduziert werden kann.

Aus reiner wirtschaftlicher oder Safety-Perspektive ist es sicherlich kein Nachteil, dass durch Automatisierung und das daraus resultierende Spannungsfeld der eigene kreative Arbeitsbeitrag der Lotsen verloren geht. Wir argumentieren aber aus einer menschenzentrierten Perspektive und weisen auf die fatalen Folgen hin, die sich durch diese restriktiven Bedingungen ergeben können, z.B. sinkender Handlungsspielraum, dadurch weniger Motivation, dadurch kann die Qualität der Arbeit sinken, was in HROs fatal wäre und folglich kann es doch schnell wieder zu einem Safety-Thema werden (vgl. auch Hancock 2020). Der Mensch sollte also auch aus Safety-Perspektive jederzeit mitgedacht werden, um weiterhin eine zuverlässige Arbeitsweise bei zunehmender Automatisierung in HROs gewährleisten zu können.

Ferner haben die Ergebnisse in Übereinstimmung mit bisherigen Studien (z.B. Parasuraman et al. 2000) einen weiteren Nebeneffekt zunehmender Automatisierung gezeigt: Die Aufrechterhaltung der weiterhin benötigten KSAOs bei einer zunehmend passiven Rolle stellt in der Praxis eine große Herausforderung dar. Dadurch, dass die Beschäftigten ihre KSAOs nicht mehr regelmäßig aktiv einbringen und somit trainieren können, resultiert die Gefahr des Fähigkeitsverlustes, was im Falle der Eingriffsnotwendigkeit, z. B. bei Systemausfall, in HROs fatale Folgen nach sich kann.

\subsection{Limitationen}

Es bleibt auf die charakteristischen Limitationen qualitativer Methoden hinzuweisen. Die Erkenntnisse repräsentieren die subjektive Wahrnehmung der Interviewten und könnten durch ihre Reflektionsfähigkeit und soziale Erwünschtheit limitiert sein. Da die Interviews auf Freiwilligkeit beruhen, ist es zudem möglich, dass sich vor allem die Personen meldeten, die dem Thema Automatisierung besonders kritisch gegenüberstehen und in den Interviews eine Möglichkeit sahen, ihren Gefühlen Ausdruck zu verleihen. Dadurch wären die negativen Auswirkungen der veränderten menschlichen Rolle zu relativieren. Ferner könnte die kleine und sehr spezifische Stichprobe einen limitierenden Faktor, vor allem bzgl. des Alters, des Geschlechts und potenzieller kultureller Unterschiede, darstellen. In Folgestudien sollten weitere HROs herangezogen werden. Es ist zudem empfehlenswert, die bisherigen qualitativen Ergebnisse durch quantitative Verfahren zu ergänzen.

In diesem Beitrag wurden vor allem die individuellen KSAOs betrachtet, obwohl in HROs vordergründig Routinen und Umgangsweisen im Team und in der Organisation für die zuverlässige Arbeitsweise verantwortlich sind (Weick und Sutcliffe 2016). Die Human Factors Forschung zeigt jedoch deutlich, dass auch den individuellen Kompetenzen ein hoher Stellenwert zukommt und jedes Teammitglied einen sicherheitskritischen Faktor einnimmt (z. B. Crameri et al. 2019; Endsley und Jones 2004; Salas et al. 2005). Ein HRT kann z.B. nicht erfolgreich zusammenarbeiten, wenn die/der Einzelne nicht über die notwendigen KSAOs verfügt. Wenn ein Teammitglied bspw. keine gutes Situationsbewusstsein hat, kann die notwendige Information im Abgleich mit dem gesamten Team nicht korrekt eingebracht werden, was fatale Folgen nach sich ziehen kann (vgl. Endsley und Jones 2004). An diesem Beispiel wird deutlich, dass auch die individuellen KSAOs entscheidend für den Erfolg einer HRO sind und deren Analyse berechtigt ist. Insgesamt dürften die Ergebnisse der vorliegenden Stu- 
die einen wesentlichen Beitrag für Wissenschaft und Praxis leisten und erste Erkenntnisse zu den veränderten Anforderungen in HROs infolge zunehmender Automatisierung liefern.

\subsection{Implikationen}

Praktische Implikationen ergeben sich vor allem für das Kompetenzmanagement und eine menschengerechte Arbeitsgestaltung. Die Ergebnisse legen nahe, dass bei zunehmender Automatisierung neben den allgemeinen Anforderungen an eine Berufstätigkeit auch das Systemwissen und -verständnis angemessen berücksichtigt werden sollten. Die Operateure müssen die Systemgrenzen und Systemlogik verstehen. Die digitale Kompetenz, also die Fähigkeit zum Umgang mit und zur Nutzung von digitalen Technologien (van Dijk 2013) ist dabei eine wichtige Voraussetzung. Es ist empfehlenswert, diese Fähigkeiten in bestehende Kompetenzmodelle aufzunehmen und durch regelmäßige Schulungen aufrechtzuerhalten. Zudem sind Schulungen zum Umgang mit hoch komplexen und volatilen Systemen zur Steigerung der mentalen Agilität (Griffith und West 2013) unabdingbar. Zeitgleich gilt, auch die Grundanforderungen in HROs durch regelmäßige Übungseinheiten trotz zunehmender Automatisierung stets manuell zu trainieren, um der erhöhten Gefahr des Fähigkeitsverlustes in der passiven Rolle entgegenzuwirken. Hierzu wären Simulationen unter geringem bis keinem Automationsgrad sinnvoll sowie eine dynamische Veränderung der Funktionsallokation zwischen Mensch und Maschine. Hierbei gilt, kritisch zu hinterfragen, ob Mensch oder System darüber entscheiden sollten, wer wann welche Funktionen ausführen darf (vgl. Manzey 2008). Diese Pflichteinheiten könnten in einem Kompetenzlogbuch (vgl. North und Reinhardt 2005) dokumentiert und im Sinne eines holistischen Kompetenzmanagementansatzes in die regelmäßigen Mitarbeitergespräche integriert werden.

Für die Arbeitsgestaltung gilt, das identifizierte Spannungsverhältnis zwischen der passiven Rolle und den gestiegenen Kompetenzanforderungen zu reduzieren. Dies könnte z.B. durch zuverlässigere Systeme oder sinnvolle Funktionsallokation (vgl. Abschn. 4) sowie durch Aufgabenerweiterung (Job Enlargement) auf ähnlichem Anforderungsniveau geschehen. So könnten zusätzliche Sonderaufgaben an Beschäftigte verteilt werden, z. B. Unterstützung bei der Ausbildung neuer Fluglotsen. Dadurch könnten für Beschäftigte neue sinnstiftende Elemente in ihrer Arbeitstätigkeit geschaffen werden, sodass trotz des Spannungsverhältnisses weiterhin aktiv die KSAOs zum Einsatz kommen und folglich Motivation und Arbeitszufriedenheit aufrechterhalten werden können. Der Mensch sollte trotz Automation ausreichend ,im Loop“ bleiben (vgl. out-of-the-loop performance problem in Endsley und
Kiris 1995), um weiterhin die Systemlogik nachvollziehen zu können, das Situationsbewusstsein stets aufrechterhalten zu können und das Gefühl zu haben, die eigenen KSAOs aktiv einbringen sowie selbst zum Arbeitsergebnis beitragen zu können. Wir empfehlen einen frühzeitigen Einbezug der Betroffenen schon während der Entwicklungsphase. So können sie aus ihrer menschenzentrierten Perspektive gestaltend Einfluss nehmen, wodurch ihnen wieder ein aktiver Part zukommt. Dies wurde in den Interviews seitens der Lotsen als besonders positiv verzeichnet.

Obgleich die präsentierten Erkenntnisse überwiegend negative Effekte der Automatisierung aus einer menschenzentrierten Perspektive darstellen, möchten wir abschlieBend auch auf die positiven Aspekte hinweisen. Nur dank der technologischen Unterstützung sind Fluglotsen in der Lage, den heutigen Verkehr in einem stark ausgelasteten Luftraum sicher und effizient zu managen. Zusammenfassend kann festgehalten werden, dass Automation kein Alles-oder-Nichts-Phänomen darstellt, sondern immer ein Abwägungsprozess zwischen den Vorteilen eines höheren Automationsgrads und den damit einhergehenden Nachteilen für die menschliche Rolle vorangestellt werden sollte.

Acknowledgment Dieser Artikel entstand im Rahmen des von der Diginomics Research Group unterstützten Forschungsprojekts „Wissenschaftliche Analyse der Auswirkungen der Automatisierung auf die Arbeitsgestaltung und die Anforderungen an Mitarbeiter in High Reliability Organizations“.

Funding Open Access funding enabled and organized by Projekt DEAL.

Open Access Dieser Artikel wird unter der Creative Commons Namensnennung 4.0 International Lizenz veröffentlicht, welche die Nutzung, Vervielfältigung, Bearbeitung, Verbreitung und Wiedergabe in jeglichem Medium und Format erlaubt, sofern Sie den/die ursprünglichen Autor(en) und die Quelle ordnungsgemäß nennen, einen Link zur Creative Commons Lizenz beifügen und angeben, ob Änderungen vorgenommen wurden.

Die in diesem Artikel enthaltenen Bilder und sonstiges Drittmaterial unterliegen ebenfalls der genannten Creative Commons Lizenz, sofern sich aus der Abbildungslegende nichts anderes ergibt. Sofern das betreffende Material nicht unter der genannten Creative Commons Lizenz steht und die betreffende Handlung nicht nach gesetzlichen Vorschriften erlaubt ist, ist für die oben aufgeführten Weiterverwendungen des Materials die Einwilligung des jeweiligen Rechteinhabers einzuholen.

Weitere Details zur Lizenz entnehmen Sie bitte der Lizenzinformation auf http://creativecommons.org/licenses/by/4.0/deed.de.

\section{Literatur}

Aepli, M., Angst, V., Iten, R., Kaiser, H., Lüthi, I., \& Schweri, J. (2017). Die Entwicklung der Kompetenzanforderungen auf dem Arbeitsmarkt im Zuge der Digitalisierung. Studie. Bern: Staatssekretariat für Wirtschaft (SECO).

Bainbridge, L. (1983). Ironies of automation. Automatica, 19(6), $775-779$. 
Becker, J.H., Ebert, H., \& Pastoors, S. (2018). Praxishandbuch berufliche Schlüsselkompetenzen. 50 Handlungskompetenzen für Ausbildung, Studium und Beruf. Berlin: Springer.

Berufenet (2019). Steckbrief Fluglotse/Fluglotsin. https://berufenet. arbeitsagentur.de/berufenet/bkb/7355.pdf. Zugegriffen: 27. Juli 2020.

Böving, H., Glaß, E., Haberzeth, E., \& Umbach, S. (2019). Digitalisierte Arbeit und menschliche Initiative. Empirische Analysen aus Logistik und Einzelhandel. In R. Dobischat, B. Käpplinger, G. Molzberger \& D. Münk (Hrsg.), Bildung 2.1 für Arbeit 4.0? (S. 141-160). Wiesbaden: Springer VS.

Bundesstelle für Flugunfalluntersuchung (2004). Untersuchungsbericht. Unfall 01. Juli 2002 nahe Überlingen/Bodensee. https:// www.bfu-web.de/DE/Publikationen/Untersuchungsberichte/2002/ Bericht_02_AX001-1-2.pdf?_blob=publicationFile. Zugegriffen: 27. Juli 2020.

Campion, M. A., Fink, A. A., Ruggeberg, B. J., Carr, L., Phillips, G. M., \& Odman, R. B. (2011). Doing competencies well: best practices in competency modeling. Personnel psychology, 64(1), 225-262.

Cascio, W.F., \& Montealegre, R. (2016). How technology is changing work and organizations. Annual Reviw of Organizational Psychology and Organizational Behavior, 3(1), 349-375.

Crameri, L., Hettiarachchi, I., \& Hanoun, S. (2019). A review of individual operational cognitive readiness: theory development and future directions. Human Factors. https://doi.org/10.1177/ 0018720819868409.

Demerouti, E. (2020). Turn digitalization and automation to a job resource. Applied Psychology: An International Review. https://doi. org/10.1111/apps.12270

DGFP (2016). Leitfaden: Kompetenzen im digitalisierten Unternehmen. Ergebnisse aus Expertenkreisen im Rahmen eines BMWigeförderten Forschungsprojekts. DGFP-Praxispapiere, Leitfaden 02/2016 (Deutsche Gesellschaft für Personalführung e. V.)..

Dworschak, B., Karapidis, A., Zaiser, H., \& Weisbecker, A. (2020). Kompetenzmanagement 4.0 - Kompetenz und Kompetenzentwicklung in einer digitalisierten Arbeitswelt. In R. Knackstedt, K. Kutzner, M. Sitter \& I. Truschkat (Hrsg.), Grenzüberschreitungen im Kompetenzmanagement. Kompetenzmanagement in Organisationen (S. 145-157). Berlin, Heidelberg: Springer.

Endsley, M. R., \& Jones, D. B. (2004). Designing for situation awareness: an approach to user-centered design (2. Aufl.). Boca Raton: CRC Press.

Endsley, M.R., \& Kaber, D. B. (1999). Level of automation effects on performance, situation awareness and workload in a dynamic control task. Ergonomics, 42(3), 462-492.

Endsley, M. R., \& Kiris, E. O. (1995). The out-of-the-loop performance problem and level of control in automation. Human Factors, 37(2), 387-394.

Eurocontrol (o.J.). About us. https://www.eurocontrol.int/about-us\# introduction. Zugegriffen: 27. Juli 2020.

Fletcher, J.D. (2004). Cognitive readiness: preparing for the unexpected. Alexandria: Institute for Defense Analyses.

Griffith, J., \& West, C. (2013). Master resilience training and its relationship to individual well-being and stress buffering among army national guard soldiers. Journal of Behavioral Health Services \& Research, 40(2), 140-155.

Grzybowska, K., \& Łupicka, A. (2017). Key competencies for Industry 4.0. Economics \& Management Innovations, 1(1), 250-253.

Hackman, J. R., \& Oldham, G. R. (1976). Motivation through the design of work: test of a theory. Organizational behavior and human performance, 16(2), 250-279.

Hagemann, V. (2011). Trainingsentwicklung für High Responsibility Teams. Lengerich: Papst Verlag.

Hagemann, V., Kluge, A., \& Ritzmann, S. (2012). Flexibility under complexity: work contexts, task profiles and team processes of high responsibility teams. Employee Relations, 34(3), 322-338.
Hammermann, A., \& Stettes, O. (2016). Qualifikationsbedarf und Qualifizierung. Anforderungen im Zeichen der Digitalisierung. IW policy paper $3 / 2016$.

Hancock, P. A. (2021). Months of monotony - moments of mayhem: Planning for the human role in a transitioning world of work. Theoretical Issues in Ergonomics Science, 22(1), 63-82.

Härtwig, C., \& Sapronova, A. (2020). Keine Angst vor der Digitalisierung! Zum Stand digitalisierter Arbeitsanforderungen in verschiedenen Industriebranchen und Tätigkeitsfeldern sowie Zusammenhänge zwischen Belastung, Ressourcen und Beanspruchungsfolgen in Deutschland. Zeitschrift für Arbeitswissenschaft. https:// doi.org/10.1007/s41449-020-00205-y

Hassenzahl, M., Diefenbach, S., \& Göritz, A. (2010). Needs, affect, and interactive products-Facets of user experience. Interacting with Computers, 22(5), 353-362.

Hauß, Y., \& Timpe, K.-P. (2000). Automatisierung und Unterstützung im Mensch-Maschine-System. In K.-P. Timpe, T. Jürgensohn \& H. Kolrep (Hrsg.), Mensch-Maschine-Systemtechnik. Konzepte, Modellierung, Gestaltung, Evaluation (S. 41-62). Düsseldorf: Symposion Publishing.

Hecklau, F., Galeitzke, M., Flachs, S., \& Kohl, H. (2016). Holistic Approach for Human Resource Management in Industry 4.0. Procedia CIRP, 54, 1-6.

Hecklau, F., Orth, R., Kidschun, F., \& Kohl, H. (2017). Human resources management: Meta-study-analysis of future competences in industry 4.0. Proceedings of the International Conference on Intellectual Capital, Knowledge Management \& Organizational Learning. (S. 163-174).

Heßler, M. (2019). Automation/Automatisierung. In K. Liggieri \& O. Müller (Hrsg.), Mensch-Maschine-Interaktion: Handbuch zu Geschichte-Kultur-Ethik (S. 235-237). Berlin: J. B. Metzler / Springer.

Hirsch-Kreinsen, H. (2018). Einleitung: Digitalisierung industrieller Arbeit. In H. Hirsch-Kreinsen, P. Ittermann \& J. Niehaus (Hrsg.), Digitalisierung industrieller Arbeit (S. 13-32). Baden-Baden: Nomos.

Hoffman, R. R., Hawley, J. K., \& Bradshaw, J. M. (2014). Myths of automation, part 2: some very human consequences. IEEE Intelligent Systems, 29(2), 82-85.

Höhne, B.P., Bräutigam, S., Longmuß, J., \& Schindler, F. (2017). Agiles Lernen am Arbeitsplatz - Eine neue Lernkultur in Zeiten der Digitalisierung. Agile Methoden, Kompetenzentwicklung, Fachkräftemangel, Digitalisierung, Industrie 4.0. Zeitschrift für Arbeitswissenschaft, 71(2), 110-119.

Kauffeld, S. (2006). Kompetenzen messen, bewerten, entwickeln. Ein prozessanalytischer Ansatz für Gruppen. Stuttgart: Schäffer-Poeschel.

Kauffeld, S., \& Paulsen, H.F.K. (2018). Kompetenzmanagement in Unternehmen. Kompetenzen beschreiben, messen, entwicklen und nutzen. Stuttgart: Kohlhammer.

LuftfahrtMagazin (2015). Österreich jetzt mit TopSky Flugsicherungssystem. https://www.luftfahrtmagazin.de/flugsicherheit/ oesterreich-jetzt-mit-topsky-flugsicherungssystem-194034.html. Zugegriffen: 27. Juli 2020.

Manzey, D.H. (2008). Systemgestaltung und Automatisierung. In P. Badke-Schaub, G. Hofinger \& K. Lauche (Hrsg.), Human Factors. Psychologie sicheren Handelns in Risikobranchen (S. 307-325). Heidelberg: Springer.

Mayring, P., \& Fenzl, T. (2014). Qualitative Inhaltsanalyse. In N. Baur \& J. Blasius (Hrsg.), Handbuch Methoden der empirischen Sozialforschung (S. 543-556). Wiesbaden: Springer.

Miller, C. A., \& Parasuraman, R. (2007). Designing for flexible interaction between humans and automation: delegation interfaces for supervisory control. Human Factors, 49(1), 57-75.

de Molina, K.-M., Kaiser, S., \& Widuckel, W. (Hrsg.). (2018). Kompetenzen der Zukunft - Arbeit 2030. Als lernende Organisation wettbewerbsfähig bleiben. Freiburg: Haufe Lexware. 
North, K. (2016). Wissensorientierte Unternehmensführung. Wissensmanagement gestalten (6. Aufl.). Wiesbaden: Springer.

North, K., \& Reinhardt, K. (2005). Kompetenzmanagement in der Praxis. Mitarbeiterkompetenzen systematisch identifizieren, nutzen und entwickeln. Wiesbaden: Gabler.

O*NET (2019). Summary report for: 53-2021.00-air traffic controllers. https://www.onetonline.org/link/summary/53-2021.00. Zugegriffen: 27. Juli 2020.

Onnasch, L. (2015). Benefits and costs of automation support: the role of function allocation and automation reliability. Dissertation. Berlin: Technische Universität.

Onnasch, L., Wickens, C.D., Li, H., \& Manzey, D. H. (2014). Human performance consequences of stages and levels of automation: an integrated meta-analysis. Human Factors, 56(3), 476-488.

Parasuraman, R., \& Manzey, D.H. (2010). Complacency and bias in human use of automation: an attentional integration. Human Factors, 52(3), 381-410.

Parasuraman, R., \& Riley, V. (1997). Humans and automation: use, misuse, disuse, abuse. Human Factors, 39(2), 230-253.

Parasuraman, R., Molloy, R., \& Singh, I.L. (1993). Performance consequences of automation-induced "complacency". The International Journal of Aviation Psychology, 3(1), 1-23.

Parasuraman, R., Sheridan, T. B., \& Wickens, C. D. (2000). A model for types and levels of human interaction with automation. IEEE Transactions on Systems, Man, and Cybernetics_part A: Systems and Humans, 30(3), 286-297.

Parker, S. K., \& Grote, G. (2020). Automation, algorithms, and beyond: Why work design matters more than ever in a digital world. Applied Psychology: An International Review. https://doi.org/10. 1111/apps.12241

Petry, T. (2019). Digital Leadership - Unternehmens- und Personalführung im digitalen Zeitalter. In T. Petry (Hrsg.), Digital Leadership. Erfolgreiches Führen in Zeiten der Digital Economy (2. Aufl. S. 23-126). Freiburg: Haufe.

Roberts, K. H. (1990). Some characteristics of one type of high reliability organization. Organization Science, 1(2), 160-176.

Roberts, K.H., \& Rousseau, D.M. (1989). Research in nearly failure-free, high-reliability organizations: having the bubble. IEEE Transactions on Engineering management, 36(2), 132-139.

Roberts, K. H., Madsen, P., Desai, V., \& Van Stralen, D. (2005). A case of the birth and death of a high reliability healthcare organisation. BMJ Quality \& Safety, 14(3), 216-220.

Ryan, R. M., \& Deci, E.L. (2000). Self-determination theory and the facilitation of intrinsic motivation, social development, and wellbeing. American Psychologist, 55(1), 68-78.

Salas, E., Sims, D. E., \& Burke, C.S. (2005). Is there a "big five" in teamwork? Small Group Research, 36(5), 555-599.

Sheridan, T. B., \& Parasuraman, R. (2005). Human-automation interaction. Reviews of Human Factors and Ergonomics, 1(1), 89-129.

Shippmann, J. S., Ash, R. A., Battista, M., Carr, L., Eyde, L. D., Hesketh, B., et al. (2000). The practice of competency modeling. Personnel psychology, 53(3), 703-740.

SKYbrary (2019). Flight progress strips. https://www.skybrary.aero/ index.php/Flight_Progress_Strips. Zugegriffen: 27. Juli 2020.

Steinke, S. (2013). Skyguide: Mausklick statt Papierstreife. https:// www.aero.de/news-18481/Skyguide-Mausklick-statt-Papier streifen.html. Zugegriffen: 27. Juli 2020.

Sutcliffe, K.M. (2011). High reliability organizations (HROs). Best Practice \& Research Clinical Anaesthesiology, 25(2), 133-144.

Umbach, S., Böving, H., \& Haberzeth, E. (2018). Kompetenzverschiebungen und menschliche Handlungsqualitäten im Digitalisierungsprozess. Ergebnisse aus Betriebsfallstudien in Einzelhandel und Logistik und Konsequenzen für die berufliche Weiterbildung. Berufsbildung in Wissenschaft und Praxis, 47(1), 16-19.
Van Dijk, J. (2013). Digitale Spaltung und digitale Kompetenzen. In A. Schüller-Zwierlein \& N. Zillien (Hrsg.), Informationsgerechtigkeit - Theorie und Praxis der gesellschaftlichen Informationsversorgung (S. 108-133). Berlin, Boston: De Gruyter.

Weick, K.E., \& Sutcliffe, K. M. (2016). Das Unerwartete managen. Wie Unternehmen aus Extremsituationen lernen (3. Aufl.). Stuttgart: Schäffer-Poeschel.

Wickens, C.D., Li, H., Santamaria, A., Sebok, A., \& Sarter, N.B. (2010). Stages and levels of automation: an integrated meta-analysis. Proceedings of the Human Factors and Ergonomics Society Annual Meeting, 54(4), 389-393.

Windelband, L., Fenzl, C., Hunecker, F., Riehle, T., Spöttl, G., Städtler, H., Hribernik, K., \& Thoben, K.-D. (2010). Abschlussbericht zur Studie „Qualifikationsanforderungen durch das Internet der Dinge in der Logistik"

Witzel, A. (2000). Das Problemzentrierte Interview. Forum: Qualitative Sozialforschung, 22(1), 1-9.

Yeh, M., Merlo, J.L., Wickens, C.D., \& Brandenburg, D.L. (2003). Head up versus head down: the costs of imprecision, unreliability, and visual clutter on cue effectiveness for display signaling. Human Factors, 45(3), 390-407.

Zajac, M., \& Schwede, C. (2017). Abschlussbericht zum Projekt QUALI 4.0 - Kompetenzentwicklungsstudie Industrie 4.0. Dortmund: Fraunhofer IML, BMBF.

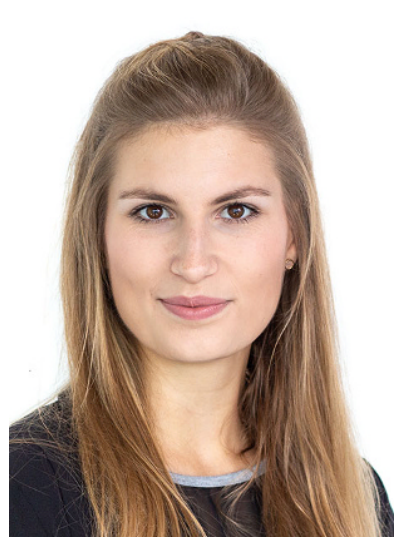

Michèle Rieth hat einen Bachelor- und Masterabschluss der Wirtschaftspsychologie und ist seit 2018 wissenschaftliche Mitarbeiterin und Doktorandin am Fachgebiet Wirtschaftspsychologie und Personalwesen der Universität Bremen. In ihrer Forschung beschäftigt sie sich vor allem mit den Auswirkungen der Automatisierung in High Reliability Organizations, insbesondere den daraus resultierenden veränderten Anforderungen an das Personal.

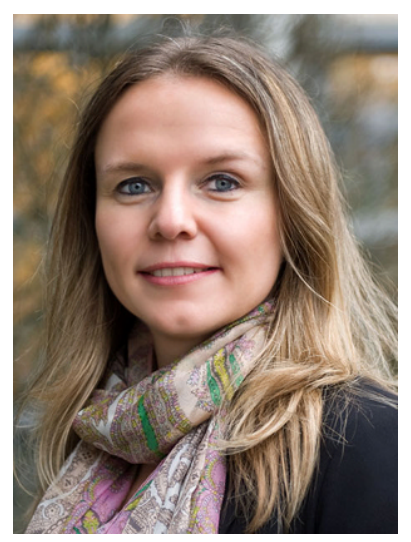

Prof. Dr. Vera Hagemann ist Leiterin des Fachgebiets für Wirtschaftspsychologie und Personalwesen an der Universität Bremen. In ihrer Forschungstätigkeit setzt sie sich in unterschiedlichen Projekten mit den Themen Kompetenzerwerb, High Responsibility Teams und Teamtraining, Evaluation, Feedback und Debriefing sowie Human Resource Analytics und Veränderungen der Arbeits- und Führungsbeziehungen sowie der Anforderungen und Ressourcen der Mitarbeitenden in sich wandelnden Arbeitskontexten aufgrund von Digitalisierung und Automatisierung auseinander. 